\title{
Endoplasmic Reticulum Stress Induces Tau Pathology and Forms a Vicious Cycle: Implication in Alzheimer's Disease Pathogenesis
}

Yuen-Shan Ho ${ }^{\mathrm{a}}$, Xifei Yang, ${ }^{\mathrm{a}, \mathrm{e}}$, Jeffery Chi-Fai Lau ${ }^{\mathrm{a}}$, Clara Hui-Ling Hung ${ }^{\mathrm{a}}$, Suthicha Wuwongse ${ }^{\mathrm{a}, \mathrm{d}}$, Qishan Zhang ${ }^{\mathrm{a}}$, Jianzhi Wang ${ }^{\mathrm{f}}$, Larry Baum ${ }^{\mathrm{g}}$, Kwok-Fai So ${ }^{\mathrm{a}, \mathrm{b}, \mathrm{c}}$ and Raymond Chuen-Chung Chang ${ }^{\mathrm{a}, \mathrm{b}, \mathrm{c}, *}$

${ }^{a}$ Laboratory of Neurodegenerative Diseases, Department of Anatomy, LKS Faculty of Medicine, The University of Hong Kong, Pokfulam, Hong Kong SAR, China

${ }^{\mathrm{b}}$ Research Centre of Heart, Brain, Hormone and Healthy Aging, The University of Hong Kong, Pokfulam, Hong Kong SAR, China

${ }^{\mathrm{c}}$ State Key Laboratory of Brain and Cognitive Sciences, The University of Hong Kong, Pokfulam, Hong Kong SAR, China

${ }^{\mathrm{d}}$ Department of Psychiatry, LKS Faculty of Medicine, The University of Hong Kong, Pokfulam, Hong Kong SAR, China

${ }^{\mathrm{e}}$ Shenzhen Centre of Disease Control and Prevention, Shenzhen, China

${ }^{\mathrm{f}}$ Department of Pathophysiology, Tongji Medical College, Huazhong University of Science and Technology, Wuhan, China

${ }^{\mathrm{g}}$ School of Pharmacy, Faculty of Medicine, The Chinese University of Hong Kong, Shatin, Hong Kong SAR, China

Handling Associate Editor: Zhizhong Guan

Accepted 25 October 2011

\footnotetext{
*Correspondence to: Dr. Raymond Chuen-Chung Chang, Department of Anatomy, Faculty of Medicine, The University of Hong Kong, 21 Sassoon Road, Pokfulam, Hong Kong SAR, China. Tel.: +852 2819 9127; Fax: +852 2817 0857; E-mail: rccchang@hku.hk.
} 


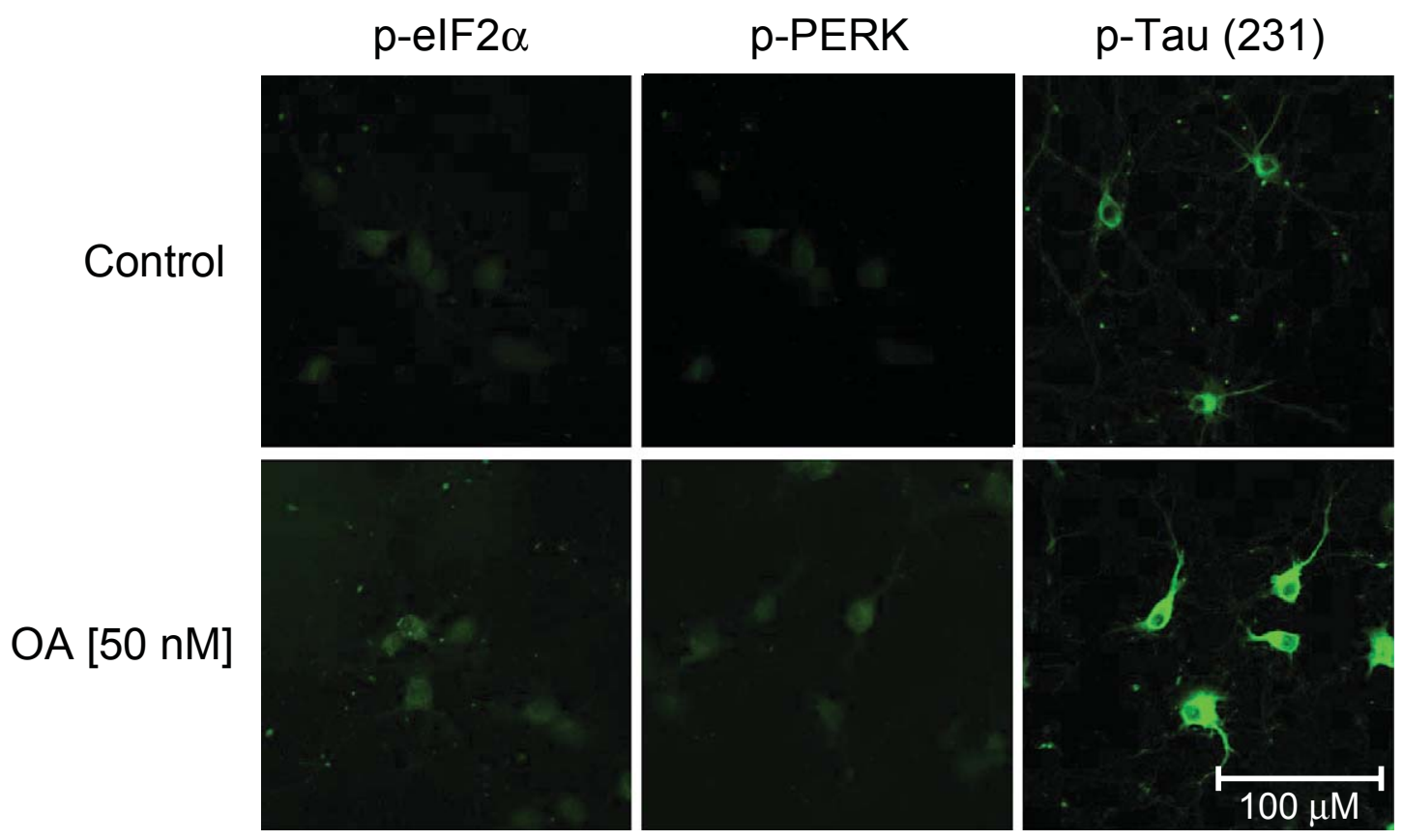

Supplementary Figure 1. Okadaic acid (OA)-induced hyperphosphorylation of tau led to endoplasmic reticulum-stress in primary cultures of hippocampal neurons. OA $(50 \mathrm{nM})$ was added to neuronal cultures and incubated for $6 \mathrm{~h}$ to induce tau hyperphosphorylation. The neurons were fixed with $4 \%$ paraformaldehyde and stained with specific antibodies against p-eIf $2 \alpha, \mathrm{p}$-PERK, and p-Tau (231). Representative photos of each group are shown, magnification $=400 \mathrm{X}$. The level of $\mathrm{p}$-Tau (231) was markedly increased in the OA-treated group. In the same time, the levels of p-eIF2 $\alpha$ and p-PERK were also increased.

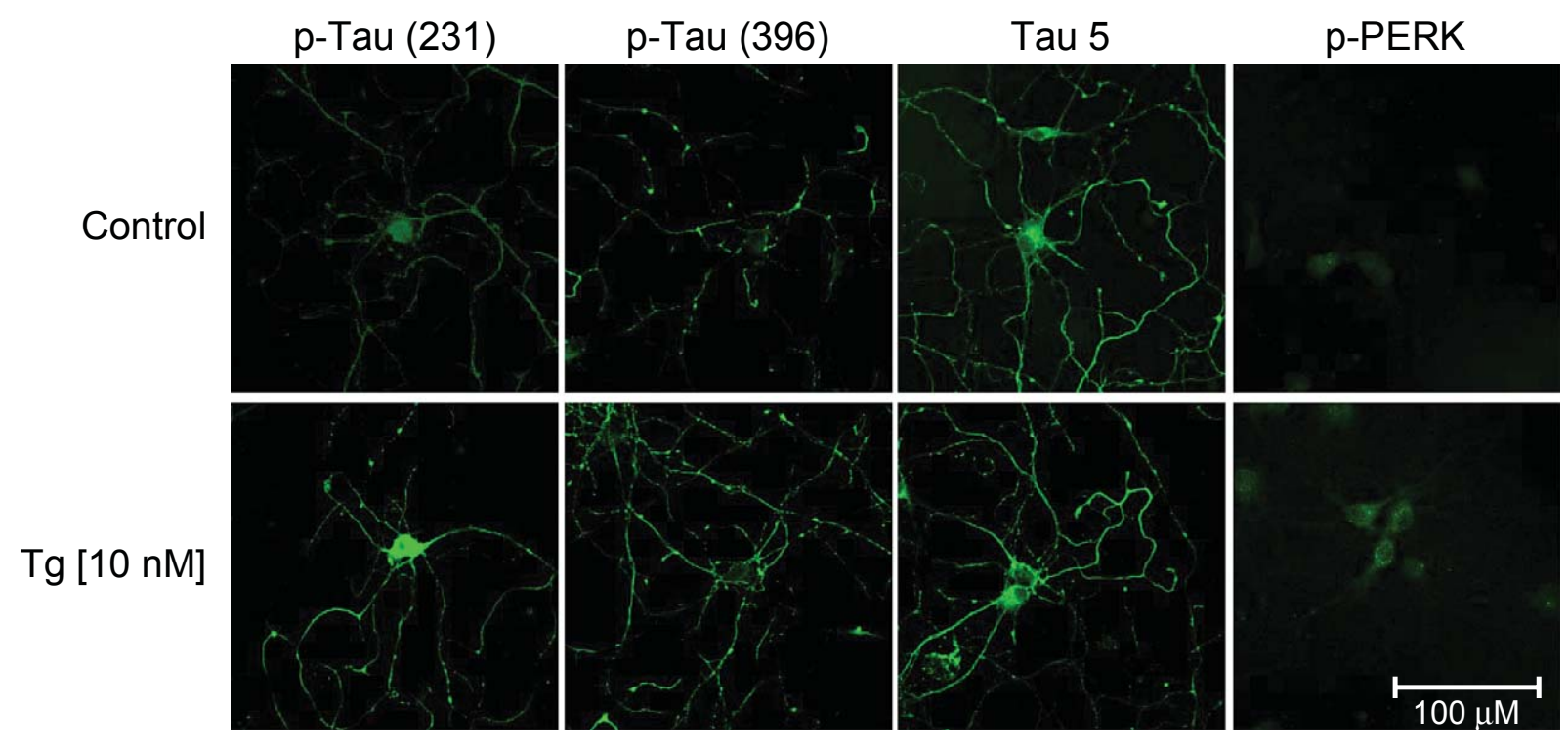

Supplementary Figure 2. Thapsigargin ( $\mathrm{Tg}$ )-induced unfolded protein response (UPR) and phosphorylation of tau in primary cultures of hippocampal neurons. Thapsigargin $(10 \mathrm{mM})$ was added to neuronal cultures and incubated for $6 \mathrm{~h}$ to induce endoplasmic reticulum-stress. The neurons were fixed with 4\% paraformaldehyde and stained with specific antibodies against p-Tau (231), p-Tau (396), Tau 5, and p-PERK. Representative photos of each group are shown, magnification $=400 X$. The level of p-PERK, a UPR marker, was elevated in the thapsigargintreated group. In the same time, the levels of p-Tau (231) and p-Tau (396) were also increased. The level of total tau, which was detected by the Tau 5 antibody, remained unchanged. 
(A)

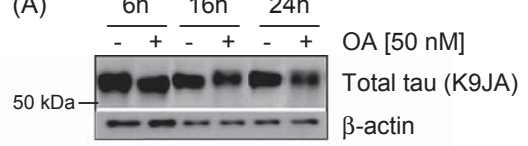

(B) $\quad 6 \mathrm{~h} \quad 12 \mathrm{~h} \quad 18 \mathrm{~h} \quad 24 \mathrm{~h}$

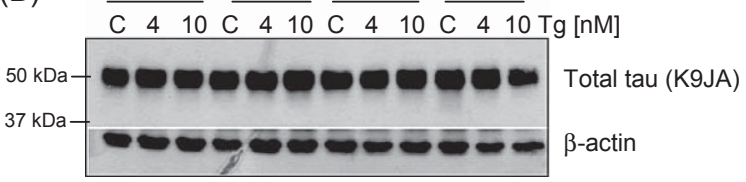

Supplementary Figure 3. Levels of total tau were further confirmed by using the K9JA antibody. A) The pattern of total tau detected by K9JA antibody in okadaic acid (OA)-treated cultures was similar to those detected by the Tau 5 antibody in Fig. 2A. B) The pattern of total tau detected by K9JA antibody in thapsigargin (Tg)treated cultures was similar to those detected by the Tau 5 antibody in Fig. 3E. 